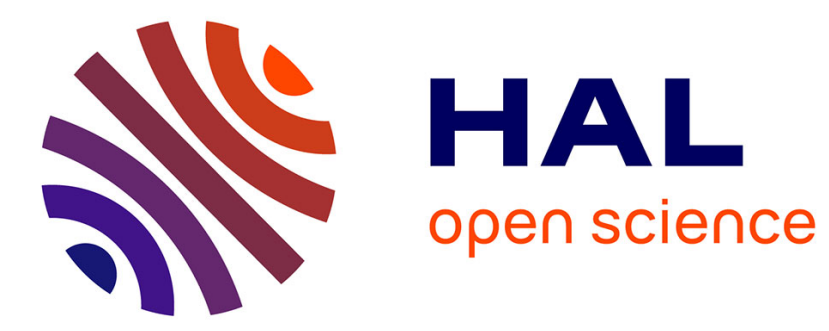

\title{
Measurement and interpretation of counterion distributions around cylindrical micelles
}

\author{
S.-L. Chang, C.-Y. Ku, S.-H. Chen, Jiali Lin
}

\section{To cite this version:}

S.-L. Chang, C.-Y. Ku, S.-H. Chen, Jiali Lin. Measurement and interpretation of counterion distributions around cylindrical micelles. Journal de Physique IV Proceedings, 1993, 03 (C8), pp.C8-117C8-127. 10.1051/jp4:1993822 . jpa-00252253

\section{HAL Id: jpa-00252253 https://hal.science/jpa-00252253}

Submitted on 1 Jan 1993

HAL is a multi-disciplinary open access archive for the deposit and dissemination of scientific research documents, whether they are published or not. The documents may come from teaching and research institutions in France or abroad, or from public or private research centers.
L'archive ouverte pluridisciplinaire HAL, est destinée au dépôt et à la diffusion de documents scientifiques de niveau recherche, publiés ou non, émanant des établissements d'enseignement et de recherche français ou étrangers, des laboratoires publics ou privés. 


\title{
Measurement and interpretation of counterion distributions around cylindrical micelles
}

\section{S.-L. CHANG, C.-Y. KU*, S.-H. CHEN* and J.S. LIN ${ }^{* *}$}

Department of Nuclear Science, National Tsing-Hua University, Taiwan, R.O.C.

* Department of Nuclear Engineering, Massachusetts Institute of Technology, Cambridge, MA 02139, U.S.A.

${ }^{* *}$ Solid State Division, Oak Ridge National Laboratory, Oak Ridge, TN, U.S.A.

\begin{abstract}
Radial distributions of heavy monovalent counterions around cylindrical micelles are measured directly using a small angle $x$-ray scattering. For the purpose of the analysis, the shape and size of the micelles are determined separately by a small angle neutron scattering. The micelles we used are formed by a comb-shaped copolymer poly-(1-octadecene-co-maleic anhydride), abbreviated as PODMA in aqueous solutions neutralized by cesium hydro-oxides. The results are used to critically test the validity of solutions of non-linear Poisson-Boltzmann (PB) equation in the cell model to predict the radial distributions of monovalent counterions. The accuracy of the solutions of the non-lineaar PB equation for different magnitudes of linear charge density parameter is checked against Monte Carlo simulations. For comparison, we also show results of a similar experiment and analysis done for the counterion distributions around persistent length DNA in solution.
\end{abstract}




\section{INTRODUCTION}

Clustering of small ions such as the counterions or salt ions of an opposite charge around surfaces of highly charged macromolecules, polymers and amphiphilic aggregates, collectively called macroions, in solution has long been theoretically predicted [1]. The resulting screening of the repulsive electrostatic interactions between macroions has a fundamental influence on the stability of the conformation of these polyelectrolytes and their colligative properties such as the osmotic pressure of the solution [2]. We shall call generally the accumulation of counterions along a highly charged macromolecular or micellar surface a "charge condensation" phenomenon regardless of whether the surface is planar, cylindrical or spherical. The basic idea is that the condensed counterions effectively neutralize an equivalent amount of the charge of the macroion so that, looking from another macroion, the complex acts as a dressed macroion of a lower surface charge density. This phenomenon has an important physical implication that, if we accept the concept of a dressed(reduced) or a renormalized charge on the macroion, then the effective interactions between macroions and macroions and counterions are greatly reduced and as a consequence, the thermodynamic and structural properties of the system may be well described by a mean field theory such as the Poisson-Boltzmann (PB) equation [3]. In this paper we shall briefly describe an experimental test of the accuracy of non-linear PB equation by directly measuring counterion radial distributions around surface of cylindrical polyelectrolytes by small angle $x$-ray scattering $[4,5,6,7]$.

\section{ION DISTRIBUTION FOR ROD-LIKE POLYIONS}

\section{A. Poisson-Boltzmann Equation}

In a cylindrical geometry, the PB equation can be solved exactly for a cell model when there is no salt present $[8,9]$. Suppose we have a rod-like macromolecule or a cylindrical micelle of length $\mathrm{L}$ and cross-sectional radius of $a$, dispersed in an aqueous solution with a number density $N_{p}$. In the cell model, we imagine a cylindrical cell of height $L$ and radius of $R$ surrounding each polyion. $R$ is chosen in such a way that the condition $\pi R^{2} L N_{p}=1$ is fulfilled. One then solve the following PB equation inside the cell:

$$
\frac{d^{2} y}{d x^{2}}+\frac{1}{x} \frac{d y}{d x}=\frac{1}{2} e^{y}
$$


where $x=\kappa r, \quad \kappa^{2}=8 \pi l B^{2} n(R), n(R)$ is the ion number density at the cell boundary and $y=-z e \phi(r) / k_{B} T$ is the reduced potential. The boundary conditions are:

(i) Choose $\phi(R)=0$, i.e. reference point at the cell boundary $R$.

(ii) $\left.\frac{d y}{d x}\right|_{\kappa R}=0 \quad$ (symmetry at the cell boundary)

(iii) $\left.\frac{d y}{d x}\right|_{\kappa a}=-\frac{2 z Z l_{B}}{a \kappa L}$ (charge conservation condition; $\mathrm{Z}$, macroion charge)

It should be noted that besides the radius of macroions $a$, the boundary condition (iii) depends on a linear charge spacing along the cylindrical axis, $L / Z$, which we shall call $b$. In the literature the dimensionless parameter $\xi=l{ }_{B} / b$ is called the linear charge density parameter. The counterion radial distribution for the case of parameter $\xi$ greater than unity is given by $[8,9,10]$ :

$$
\frac{n(r)}{n(R)}=e^{y}=\left\{\frac{2|\lambda|}{\kappa r \cos \left\lfloor\lambda \mid n\left(r / R_{M}\right)\right.}\right\}^{2}
$$

The parameter $\lambda$ is the first integration constant which can be obtained by solving a transcendental equation

$$
\lambda \ln (a / R)=\frac{1}{\lambda} \tan ^{-1}\left(\frac{1}{\lambda}\right)+\frac{1}{\lambda} \tan ^{-1}\left(\frac{z \xi-1}{\lambda}\right)
$$

and compute $n(R)$ from an equation $8 \pi z^{2} l B^{n(R) R^{2}=4\left(1+\lambda^{2}\right)}$. The parameter $R_{M}$ is the second integration constant and is given by

$$
R_{M}=\operatorname{aexp}\left[\frac{1}{\lambda} \tan ^{-1}\left(\frac{z \xi-1}{\lambda}\right)\right]
$$

Using ion distribution given in Eq.(4) one can now calculate the fraction of ions $F(r)$ that lie in-between radii $(a, r)$ with a result

$$
F(r)=\frac{1}{z \xi} \lambda \tan \left[\lambda \ln \left(r / R_{M}\right)\right]+1-\frac{1}{z \xi}
$$

From this expression we see the physical meaning of $R_{M}$. The fraction of ions contains within this radius, $F\left(R_{M}\right)=1-1 / z \xi$, is the so called Manning fraction[10, 11]. In a phenomenological theory proposed by Manning [12] in 1969, he proposed that the onset of charge condensation is when the linear charge spacing $b$ decreases to a value equal or less than Bjerrum length $l_{B}$, namely, when $\xi=1$ or more. The condensed fraction is then equal to $1-1 / z \xi$. We have shown [5] 
that this definition of the condensed fraction is not always realistic, especially when $\xi$ is large.

\section{B. Accuracy of Poisson-Boltzmann Equation for Cylindrical Geometry}

A Monte Carlo simulation model which was developed by Le Bret and Zimm [13] is used to calculate the ion distribution around cylindrical polyelectrolytes in aqueous solution to check the accuracy of $\mathrm{PB}$ equation. In this model, the DNA-like polyelectrolyte is simulated as a impenetrable cylinder having discrete charges located either along the cylindrical axis or on more realistic double helical position. The long range interaction of the mobile counterions is given by a simple coulomb force, and the short-range interaction is treated as a hard sphere repulsion. Since the length of the sampling space is finite, four replica along the z-axis, two immediately above and two below, are used for calculating the potential energy to take into account the end effect. The Metropolis sampling method is applied to generate the configuration. In general, $5 \times 10^{4}$ trial moves with larger step size need to be made before the system reaches equilibrium state. Step size $\Delta h$ is chosen by trial and error so that half of the trial moves are rejected. Typically, $2 \times 10^{5}$ moves are made after the equilibrium. With the histogram that we build, the radial distribution of the counterions and co-ions per unit volume can be calculated.

The MC simulations of DNA-like polyelectrolyte, where $b=1.7 \AA$, with cylindrical radius $a=10 \AA$ and cell radius $R=100 \AA$ of two different models of phosphate group $\mathrm{PO}_{4}^{-}$locations for the system having monovalent counterions of radius $1 \AA$ and $3 \AA$ at salt free condition are shown in Fig.1. It is found that the counterion radial distribution is very similar regardless of whether the phosphate groups are modeled along the cylindrical axis or on the double helices. The comparison

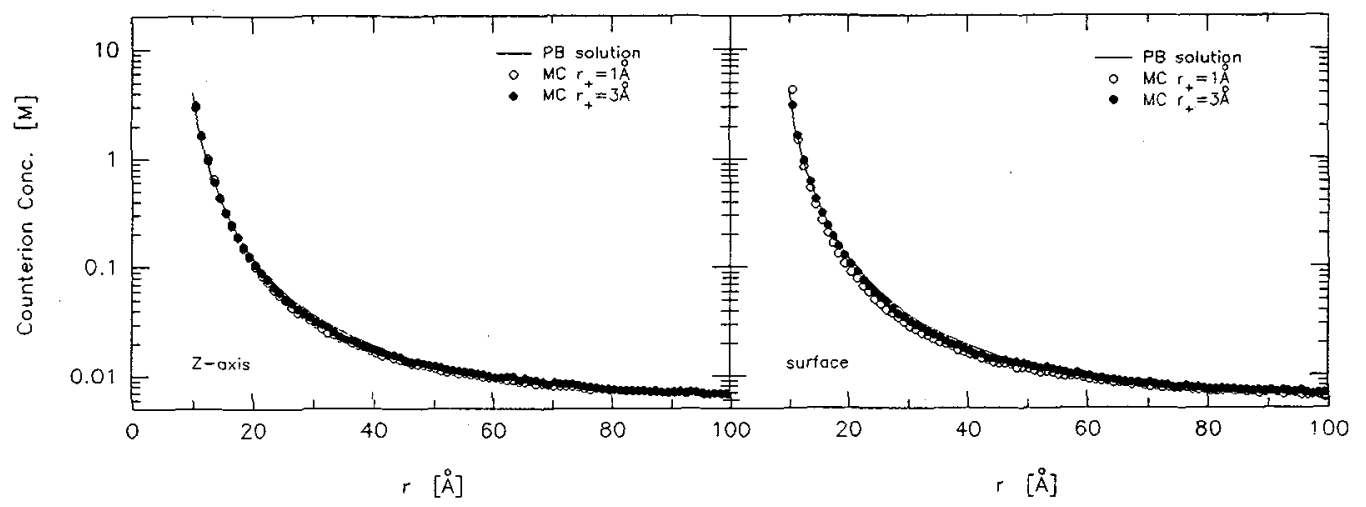

Fig.1 The comparison of the monovalent counterion distribution obtained from the PB equation and the MC simulation of the different model of the phosphate group locations. 


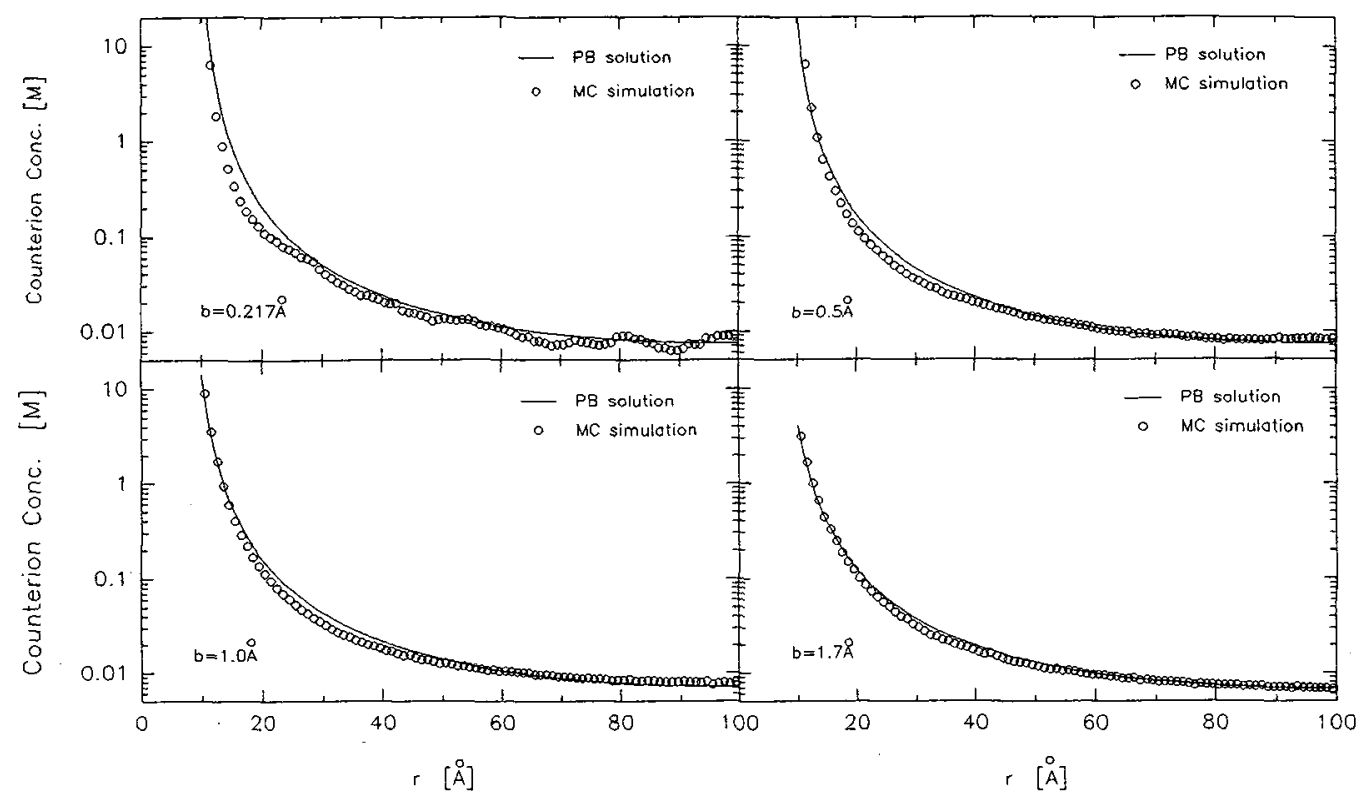

Fig.2 The comparison of the monovalent counterion distribution obtained from the PB equation and the MC simulation around a single DNA-like polyelectrolyte in a cell of $\mathrm{R}=100 \AA$ for the different linear charge spacing, $b=0.217,0.5,1.0,1.7 \AA$.

of the PB solution and the simulation results shows that the PB equation can give fairly accurate result under this condition. We made a further test to check the accuracy of $P B$ equation for different charge densities. The radial distributions of monovalent counterions of different linear charge spacing, $b=0.217,0.5,1.0,1.7 \AA$, with $a=10 \AA$ and $R=100 \AA$ are plotted in Fig.2. The size of the counterions is chosen to be $1 \AA$ in these simulations. It is shown in the figure that the discrepancy increases as $b$ decreases. This is due to the neglect of short-range repulsion among the counterions in the PB equation. Since when $b$ is smaller, the counterion concentration near the surface becomes larger, the effect of short-range repulsion becomes more significant. In Fig.3, we can see that for the PODMA-like polyelectrolyte (which will be discuss later), whose linear charge spacing $b=0.217 \AA$, with cylindrical radius $a=27 \AA$ and cell radius $R=200 \AA$ of two different models of charge group locations, on the surface or along the z-axis, for the system having monovalent counterions of radius $r_{+}=3 \AA$ (this is the hydration radius of ion), a second peak appears at $r \approx 33 \dot{A}$. The same phenomenon is also observed for DNA-like polyelectrolyte [13] but with a much larger radius of $10 \AA$. The discrepancy between $\mathrm{PB}$ solution and MC simulation is even larger in this case, where PB solution overestimates the concentration near the surface but 


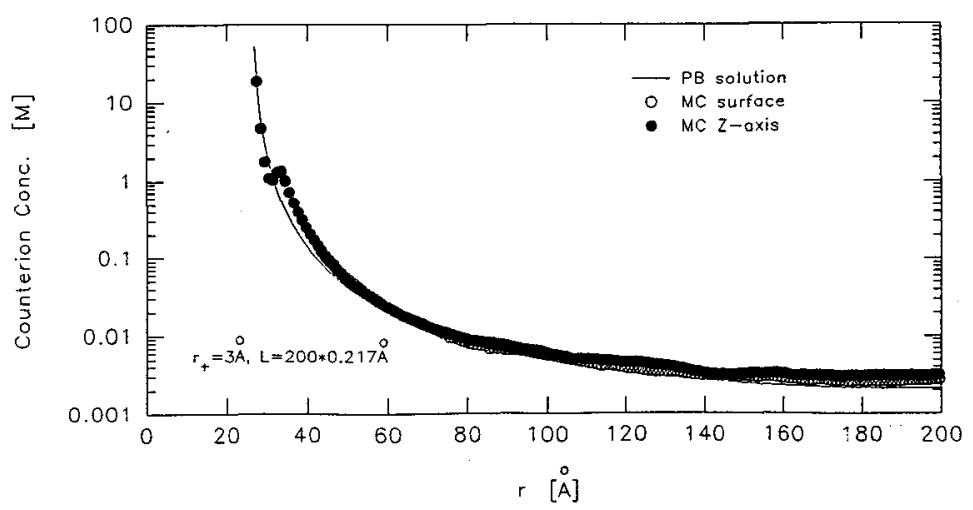

Fig.3 The comparison of the monovalent counterion distribution obtained from the $\mathrm{PB}$ equation and the MC simulation of the different model of the charge group locations for a PODMA-like polyelectrolyte in a cell model of $a=27 \AA$ and $R=200 \AA$.

underestimates it in the large $\mathbf{r}$ region.

\section{SAXS INTENSITY FROM A ROD-LIKE POLYION}

Consider a polyelectrolyte solution with $N_{p}$ macromolecules or surfactant aggregates per unit volume. The scattering intensity per unit volume (in unit of $1 / \mathrm{cm}$ ) is given by $I(Q)=N_{p} P(Q)$, where $P(Q)$ is the structure factor of a single macromolecule or aggregate. Here we do not consider the interference factor or the so called inter-particle structure factor because the solution is assumed to be sufficiently dilute. Even though in some cases the inter-particle structure factor may not be negligible because of a long-ranged electrostatic interaction, we choose not to discuss it because from the point of view of counterion distribution, the relevant quantity is the $P(Q)$ function. We denote by $Q$ the Bragg wave number for the scattering, $(4 \pi / \lambda) \sin (\theta / 2)$, where $\theta$ is scattering angle. It is physically more interesting to rewrite the intensity as

$$
I(Q)=I_{o} \tilde{P}(Q)
$$

Where $I_{o}$ is the intensity at $Q=0$ and $P(Q)$ is a normalized particle structure factor equal to unity at that point. In this paper we only give expressions for the scattering from distribution of counterions. Here is the expressions for $I_{0}$. 


$$
I_{o}=N_{p} r_{o}^{2} Z_{c}^{2} X_{c}^{2} Z^{2}
$$

In above equations, $N_{p}$ is the number of rod-like polyions per unit volume respectively, $r_{o}$, classical radius of an electron, $Z_{c}$, the atomic number of the counterion, $Z$, the total number of charge on the polyion and $X_{c}$ is a correction factor arising from consideration of solute excluded solvent molecules. In our specific case, we have counterions in water, so that denoting by subscripts $c$ and $w$ the counterion and water respectively,

$$
X_{c}=1-\left(\frac{Z}{Z_{c}}\right)\left(\frac{{ }^{c}}{v_{w}}\right) \quad(Z, \text { atomic no., } v, \text { molecular volume })
$$

For the normalized structure factors, we have first to calculate the orientation dependent form factor for a rod-like particle

$$
F(Q, \mu)=\frac{1}{Z}\left[\frac{\sin (Q \mu L / 2)}{(Q \mu L / 2)}\right] \int_{0}^{R} d r 2 \pi r L\left[n_{c}(r)-n_{c}(0)\right] J_{0}\left(Q r \sqrt{1-\mu^{2}}\right)
$$

where one is to use the ion distribution given in Eq.(4) in the integral, and then calculate by an orientational average of the square of the form factor,

$$
\tilde{P}(Q)=\int_{0}^{1} d \mu|\tilde{F}(Q, \mu)|^{2}
$$

\section{COMPARISON WITH SAXS EXPERIMENTS}

Small angle X-ray scattering experiments were performed at the 10-m SAXS instruments in the solid state division of Oak Ridge National Laboratory. The X-ray source was a $12 \mathrm{KW}$ rotating anode $\mathrm{X}$-ray generator with a $\mathrm{Cu}$ target. The characteristic $\mathrm{X}$-ray wavelength $\lambda=1.542 \AA$ was selected by a pyrolitic graphite monochromator. The source to sample distance was $3.126 \mathrm{~m}$, interrupted by a series of pinholes resulting in a beam size at the sample of $1 \mathrm{~mm}$ in diameter. The sample to detector distance was fixed at $1.7 \mathrm{~m}$. Coupled with a $2-\mathrm{D}$ position-sensitive detector of $20 \times 20 \mathrm{~cm}^{2}$ active area and $64 \times 64$ pixels, the range of Bragg wave vector $Q$ covered in the measurements was from $0.01 \AA^{-1}$ to $0.3 \AA^{-1}$. The sample was contained in a copper sample holder having two Kapton windows of thickness $50 \mu \mathrm{m}$. The sample path length was $1 \mathrm{~mm}$. The scattering signal from each sample was collected for about 6 hours. The raw data were corrected 
for instrumental noise, radiation background and water sample cell scattering. The detector sensitivity was calibrated by using a ${ }^{55} \mathrm{Fe}$ radioactive $\mathrm{X}$-ray source. The normalization to the absolute intensity was made with a sheet of polyethylene of known scattering cross section.

Since the contribution from the counterions to the scattering intensity is proportional to $\mathrm{Z}^{2}$, SAXS intensities of DNA samples with light counterions are mainly due to the DNA core. We, therefore, use NaDNA as a benchmark experiment to test the consistency of the parameters given by literature for the study of DNA. A value of DNA dry specific volume s.v.=0.57, which is given by Luzzati et al.[14], together with $\mathrm{Z}_{\mathrm{DNA}}=320$ and $\mathrm{M}_{\mathrm{DNA}}=614$ are used to calculate the scattering intensity. The length of the B-DNA fragments is $496.4 \mathrm{~A}$ and the helical diameter is taken to be $20 \AA$. The comparison of the SAXS experiment for the case of NaDNA sample with $5.4 \mathrm{mg} / \mathrm{ml}$ concentration in the $10 \mathrm{mM} \mathrm{NaCl}$ solution and the the theoretical calculation is shown in Fig.4. Symbols are the experimental data; the dashed line represents the scattering intensity due to the DNA core only; and the solid line represents the intensity due to both the DNA core and the counterions, where the counterion distribution is obtained from PB equation. Judging from the agreement between the experimental data and the calculated results, we can conclude that the choice of the specific volume and the helical diameter is appropriate. We also see from the figures that the contribution from $\mathrm{Na}^{+}$to the scattering intensity is indeed negligible, and theaddition of small amount of salt does not effect the DNA conformation.

A sample with B-DNA concentration of $5.55 \mathrm{mg} / \mathrm{ml}$ and $10 \mathrm{mM} \mathrm{CsCl}$ salt added solution is chosen to present the case of CsDNA. Since the $\mathrm{Z}$ value of $\mathrm{Cs}^{+}$is much larger than $\mathrm{Na}^{+}$, the

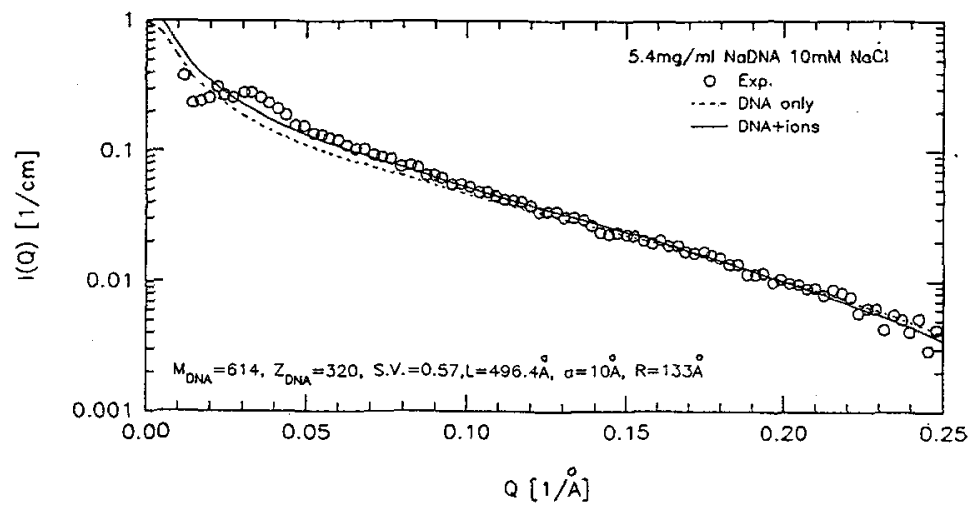

Fig.4 SAXS scattering intensity distribution for the $5.4 \mathrm{mg} / \mathrm{ml}$ NaDNA solution with $10 \mathrm{mM}$ $\mathrm{NaCl}$ added case. The counterion distribution is obtained from the $\mathrm{PB}$ equation. 

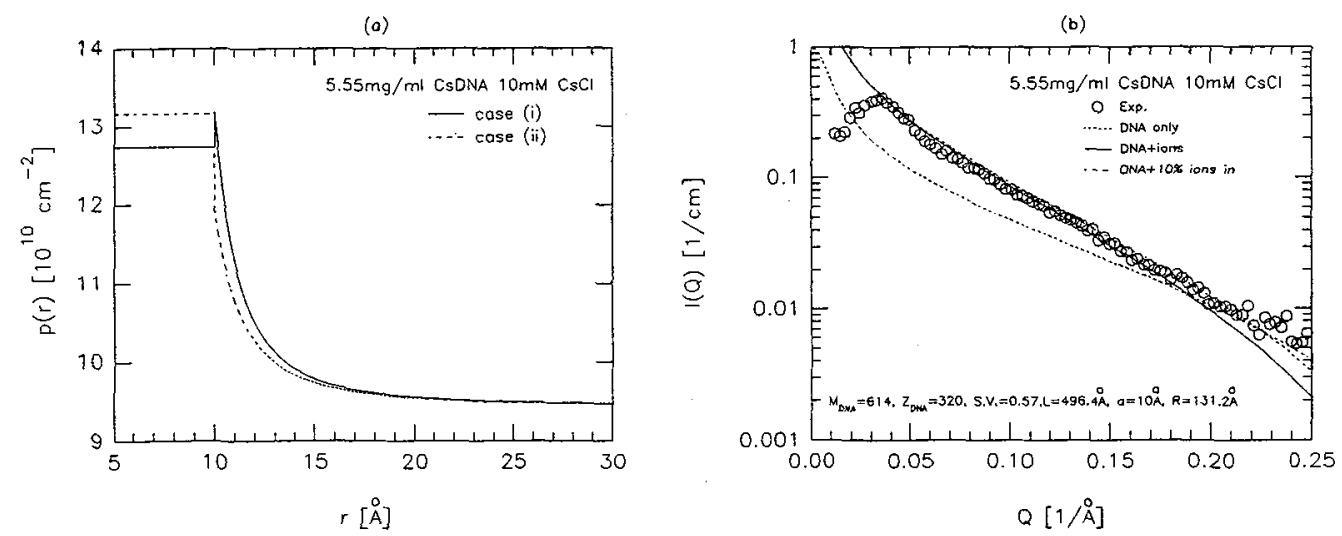

Fig.5 (a) Scattering length density distribution for a single persistence length B-DNA in a cell of $\mathrm{R}=131.2 \AA$. Solid line represents the case (i) which assumes no penetration of counterion into the core. Dotted line represents the case (ii) which assumes $10 \%$ of the counterions is inside the core. (b) The comparison of the experimental and calculated SAXS intensities for $5.55 \mathrm{mg} / \mathrm{ml} \mathrm{CsDNA}$ solution with $10 \mathrm{mM} \mathrm{CsCl}$ added.

scattering intensity from $\mathrm{Cs}^{+}$ions become detectable. However, due to the presence of the major and minor grooves in the double helical structure of B-DNA, it is practical to assume that part of the counterions penetrate into the DNA core in the cell model of PB equation. We thus consider two cases: (i) no penetration of counterions; (ii) $10 \%$ of the counterions is inside the core. The scattering length density distribution for these two cases are given in Fig.5(a), the corresponding calculated scattering intensity distributions and their comparison with the experimental data are given in Fig.5(b). It is clear in the figure that with assuming the ion penetration, the agreement between the theory and experiment becomes better for the large Q portion. Since the system is not infinite dilute, the discrepancy in the small $Q$ portion is owing to the neglect of the inter-particle structure factor $S(Q)$ in the theory.

Since the electron density of DNA core is comparable to that of heavy counterions near the surface, which is an unfavorable case for SAXS, we extend our study for the counterion distributions around cylindrical polyelectrolytes by using a cylindrical micelles formed by a comb-shaped copolymer poly[1-octadecene-co-(maleic-anhydride)] (PODMA) neutralized by $\mathrm{Cs}^{+}$in aqueous solution. This copolymer has an array of hydrophobic bydrocarbon side chain and two hydrophilic carboxylic acid head groups per repeating unit. From previous SANS studies [15], the cylindrical shape of the micelles formed by PODMA in the fully neutralized state in aqueous 

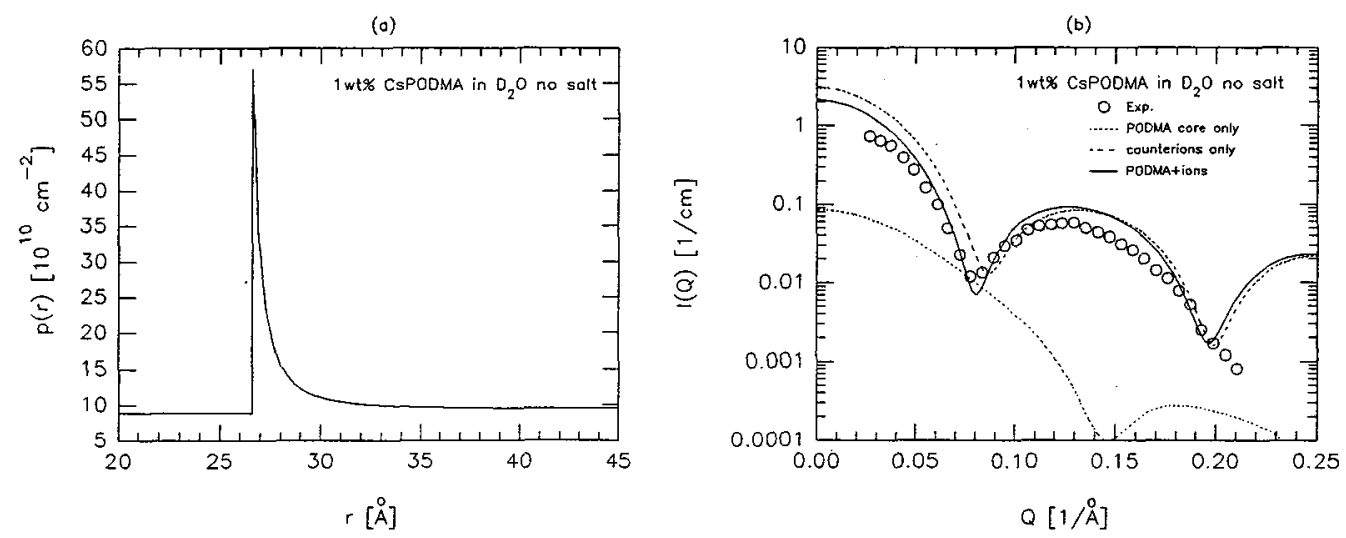

Fig.6 (a) Scattering length density distributions for a single PODMA micelle enclosed in a cell of radius $R=200.8 \AA$. (b) The comparison of the experimental and calculated SAXS intensities for 1wt\% PODMA in aqueous solution.

solution is confirmed. The electron density of this cylindrical micelle is nearly equal to that of the solvent, therefore, the major contribution to the scattering intensity is due to the heavy counterions. The number of monomers in a micellar aggregate is found to be, $P N=226$, the length of the cylindrical micelle is, $L=98.1 \AA$, and the micellar radius, $a=26.6 \AA$ [15]. From these figures and taking into account the fact that there are two $\mathrm{COO}^{-}$groups per monomer, we can get the linear charge spacing $b=98.1 /(2 \times 226)=0.217 \AA$ resulting in the linear charge density $\xi=l, b=33$. The concentration of PODMA that we used is $1 \mathrm{wt} \%$ corresponding to the cell boundary $R=201 \AA$. The conventional $\mathrm{PB}$ equation is used to compute the counterion distribution outside the micellar core. Fig.6(a) depicts the scattering length density profile of the cell. It is interesting to note that, the scattering length density of the PODMA core, $\rho_{\text {core }}=8.88 \times 10^{10} \mathrm{~cm}^{-2}$, is nearly matched out by the bulk solution, $\rho_{\mathrm{b}}=9.38 \times 10^{10} \mathrm{~cm}^{-2}$; and on the micellar surface, the scattering length density due to the accumulation of $\mathrm{Cs}^{+}$ions reaches a value of $5.71 \times 10^{11} \mathrm{~cm}^{-2}$, corresponding to the $\mathrm{Cs}^{+}$concentration $53.4 \mathrm{M}$. This is an extremely favorable condition for using SAXS to study the ion distribution around the cylindrical polyelectrolytes in solution, because the scattering intensity is mainly due to the counterions. The comparison of SAXS data with the theoretical calculation of the scattering intensity distribution in absolute scale is shown in Fig.6(b) Circles represent the experimental data, dotted line represents the scattering intensity due to the PODMA core only, dashed line that from counterions only, and solid line is the coherent sum of the above two contributions. It is impressive that the theory predicts the minimum correctly. 
However, both in the intermediate and small $Q$ ranges the theory overestimates the scattering intensity. Under the extremely high counterion concentration, $53.4 \mathrm{M}$ on the micellar surface as predicted by the PB equation, the short-range repulsion, which is completely neglected in the PB equation, should play an important role. It is strongly suggested that the PB equation overestimates the counterion accumulation near the PODMA micellar surface which is predicted by $\mathrm{MC}$ comparison as shown in Fig.3.

\section{CONCLUDING REMARKS}

We have used an exact solution of the non-linear PB equation in the cell model for the case of a cylindrical polyion surrounded by its own counterion to interpret SAXS data from persistence length DNA fragment and cylindrical micelles PODMA in solutions. It has been shown first that the contribution of the counterion, especially a heavy counterion $\mathrm{Cs}^{+}$is significant for a quantitative interpretation of SAXS data. In the case of $496 \AA$ persistence length DNA fragments where the linear charge density parameter is 4.2 the agreement between the theory and the experiment is satisfactory. However, in the case of $98 \AA$ PODMA cylindrical micelles where the linear charge density parameter is 33 , the discrepancy between the theory and the experiment is visible. This trend agree with our MC simulation results. For a decisive test of the counterion distribution, it is necessary that the counterion contribution be a dominant factor in the scattering intensity. It is therefore reasonable to expect that an anomalous $\mathrm{X}$-ray scattering technique would be appropriate in the case of DNA. The experiment for this case is in progress.

Research work of the MIT group is supported by a grant from US DOE material sciences programs.

\section{REFERENCES}

1. Oozawa,F. (1971). Polyelectrolytes. Dekker, New York.

2. Manning,G.S. (1979). Acc. Chem. Res. 12, 443.

3. Fixman,M. (1979). J. Chem. Phys. 70, 4995 .

4. Wu, C.F., Chen, S.H., Shih, L.B. and Lin,J.S. (1988). J. Appl. Cryst. 21, 853

5. Wu, C.F., Chen, S.H., Shih, L.B. and Lin,J.S. (1988). Phys. Rev. Lett. 61, 645.

6. Chang,S.L., Chen, S.H., Rill, R.L. and Lin,J.S.(1990). J. Phys. Chem. $\mathbf{9 4}, 8025$.

7. Chang,S.L., Chen, S.H., Rill, R.L. and Lin,J.S.(1991). Progr. Colloid Polym. Sci. $84,409$.

8. Alfrey, T., Berg,P.W. and Morawetz, H. (1951). J. Polym. Sci. $\underline{7}, 543$.

9. Fuoss, R.M., Katchalsky, A. and Lifson, S. (1951). Proc. Nation. Acad. Sci. 37, 579.

10. Le Bret, M. and Zimm, B.H. (1984). Biopolymers $\underline{\mathbf{2 3}}, 287$.

11. Zimm, B.H. and Le Bret, M. (1983). J. Biomol. Structure and Dynamics, $\rightarrow 461$.

12. Manning, G.S. (1969). J. Chem. Phys. $\underline{51}, 924 ; \underline{52}, 3249$.

13. Le Bret, M. and Zimm, B.H. (1984). Biopolymers $23,271$.

14. Luzzati, V., Nicolaieff, A. and Masson, F. (1961). J. Mol. Biol. 3, 185(1961); Luzzati, V.,

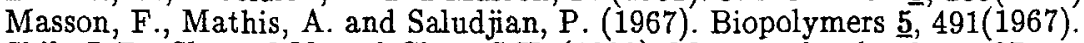

15. Shih, L.B., Sheu, E.Y. and Chen, S.H. (1988). Macromolecular 21, 1387. 